\title{
Remarks on the Unimodular Fourier Multipliers on $\alpha$-Modulation Spaces
}

\author{
Guoping Zhao, ${ }^{1}$ Jiecheng Chen, $^{2}$ and Weichao Guo ${ }^{3}$ \\ ${ }^{1}$ Department of Mathematics, Zhejiang University, Hangzhou 310027, China \\ ${ }^{2}$ Department of Mathematics, Zhejiang Normal University, Jinhua 321004, China \\ ${ }^{3}$ Department of Mathematics, Xiamen University, Xiamen 361005, China
}

Correspondence should be addressed to Weichao Guo; weichaoguomath@gmail.com

Received 15 April 2014; Revised 28 June 2014; Accepted 28 June 2014; Published 17 July 2014

Academic Editor: Yuri Latushkin

Copyright (C) 2014 Guoping Zhao et al. This is an open access article distributed under the Creative Commons Attribution License, which permits unrestricted use, distribution, and reproduction in any medium, provided the original work is properly cited.

We study the boundedness properties of the Fourier multiplier operator $e^{i \mu(D)}$ on $\alpha$-modulation spaces $M_{p, q}^{s, \alpha}(0 \leq \alpha<1)$ and Besov spaces $B_{p, q}^{s}\left(M_{p, q}^{s, 1}\right)$. We improve the conditions for the boundedness of Fourier multipliers with compact supports and for the boundedness of $e^{i \mu(D)}$ on $M_{p, q}^{s, \alpha}$. If $\mu$ is a radial function $\phi(|\xi|)$ and $\phi$ satisfies some size condition, we obtain the sufficient and necessary conditions for the boundedness of $e^{i \phi(|D|)}$ between $M_{p_{1}, q_{1}}^{s_{1}, \alpha}$ and $M_{p_{2}, q_{2}}^{s_{2}, \alpha}$.

\section{Introduction}

Let $\mathscr{F}$ and $\mathscr{F}^{-1}$ denote the Fourier transform and the inverse Fourier transform, respectively. For a bounded function $m$, the Fourier multiplier operator associated with $m$ is defined by

$$
T_{m}(f)(x)=m(D) f=\mathscr{F}^{-1}(m \mathscr{F} f)
$$

on all Schwartz functions $f \in \mathcal{S}\left(\mathbb{R}^{n}\right)$, where $m$ is called the symbol or multiplier of $T_{m}$. Fourier multipliers arise naturally from the formal solution of linear partial differential equations and from the summabilities of Fourier series. The boundedness properties of a Fourier multiplier in various function or distribution spaces contribute an important research topic in harmonic analysis, as well as many significant applications in partial differential equations.

Let $X$ and $Y$ be two function/distribution spaces with norms (or quasinorm) $\|\cdot\|_{X}$ and $\|\cdot\|_{Y}$, respectively. A bounded function $m$ is called a Fourier multiplier from $X$ to $Y$, if there exists a constant $C>0$ such that

$$
\left\|T_{m}(f)\right\|_{Y} \leq C\|f\|_{X}
$$

for all $f \in \mathcal{S}\left(\mathbb{R}^{n}\right)$. We use the above definition to avoid the situation where $\mathcal{S}\left(\mathbb{R}^{n}\right)$ is not dense in $M_{p, q}^{s, \alpha}$ when $p=\infty$ or $q=\infty$.
In this paper, we will study the unimodular Fourier multipliers on the $\alpha$-modulation space $M_{p, q}^{s, \alpha}(\alpha \in[0,1])$ (see Section 2 for the definition of $M_{p, q}^{s, \alpha}$ ). Particularly, we will focus on the unimodular Fourier multipliers with symbol $e^{i \mu(\xi)}$ for real-valued functions $\mu$. These multipliers arise when one solves the Cauchy problem for some dispersive equations. For example, for the Cauchy problem of (linear) Klein-Gordon equations

$$
\begin{gathered}
u_{t t}+(I-\Delta) u=0, \\
u(0, x)=u_{0}, \\
u_{t}(0, x)=u_{1},
\end{gathered}
$$

$(t, x) \in \mathbb{R} \times \mathbb{R}^{n}$, the formal solution is given by

$$
u(t, x)=K^{\prime}(t) u_{0}+K(t) u_{1},
$$

where

$$
K(t)=\frac{G(t)-G(-t)}{2 i\left(1+|D|^{2}\right)^{1 / 2}}
$$

and the Klein-Gordon semigroups are defined by

$$
G(t)=e^{i t\left(1+|D|^{2}\right)^{1 / 2}} \text {. }
$$


The modulation spaces were introduced by Feichtinger [1] in 1983 by the short-time Fourier transform. Now, people have recognized that the modulation spaces are very important function spaces, since they play more and more significant roles not only in harmonic analysis, but also in the study of partial differential equations. On the other hand, Besov space $B_{p, q}^{s}$ is also a popular working frame in harmonic analysis and partial differential equations. In 1992, Gröbner introduced the $\alpha$-modulation space $M_{p, q}^{s, \alpha}$ [2], that is an intermediate space between these two types of spaces with respect to the parameters $\alpha \in[0,1]$. Modulation spaces are special $\alpha$-modulation spaces in the case $\alpha=0$, and the (inhomogeneous) Besov space $B_{p, q}^{s}$ can be regarded as the limit case of $M_{p, q}^{s, \alpha}$ as $\alpha \rightarrow 1$ (see [2]). So, for the sake of convenience, we can view the Besov spaces as special $\alpha$-modulation spaces and use $M_{p, q}^{s, 1}$ to denote the inhomogeneous Besov space $B_{p, q}^{s}$.

It is known that $e^{i|D|^{\beta}}$ is not bounded on any Lebesgue space $L^{p}$ and Besov spaces $B_{p, q}^{s}$, except for $p=2$ or $\beta=1$ and $n=1$, (see $[3,4])$. However, $e^{i|D|^{\beta}}$ is bounded on the modulation space $M_{p, q}^{s}=M_{p, q}^{s, 0}$ for all $1 \leq p, q \leq \infty$, $s \in \mathbb{R}$ (see Bényi et al. [5]). Hence, the modulation spaces play an alternative role in the study of unimodular Fourier multipliers. In [5], the authors proved that if $0 \leq \beta \leq 2$, $e^{i|D|^{\beta}}$ is bounded on $M_{p, q}^{s}$ for all $1 \leq p, q \leq \infty, s \in \mathbb{R}$. Furthermore, in the case $\beta>2$, Miyachi et al. [6] showed that, for $1 \leq p, q \leq \infty$ and $s_{1}, s_{2} \in \mathbb{R}, e^{i|D|^{\beta}}$ is bounded from $M_{p, q}^{s_{1}}$ to $M_{p, q}^{s_{2}}$ if and only if $s_{1}-s_{2} \geq(\beta-2) n|1 / p-1 / 2|$. The reader also can see [7-11] for more results in this topic.

Since the $a$-modulation space $M_{p, q}^{s, \alpha}$ is an extension of the classical modulation space and it is a natural bridge connecting the modulation spaces and the Besov spaces (see $[12,13])$, in a recent paper [14], we study the boundedness of $e^{i \mu(D)}$ on function spaces $M_{p, q}^{s, \alpha}$ and establish a sufficient and necessary boundedness theorem by assuming that $\mu$ is a homoge-nous function. Thus, it will be interesting to study $e^{i \mu(D)}$ when $\mu$ is not a homogenous function. This motivates us to seek some sharp condition to ensure the boundedness on $M_{p, q}^{s, \alpha}$ for the unimodular multiplier $e^{i \mu(D)}$ when $\mu$ is not homogenous. In this note, we will focus on the case that $\mu$ is a radial but not homogeneous function. We remark that, for a radial function $\mu$, the operator $e^{i \mu(D)}$ not only is a generation for the Schrödinger semigroup $e^{i|D|^{\beta}}$, but also works for the KleinGordon semigroup with symbol $e^{i \mu}$, where $\mu(\xi)=\left(1+|\xi|^{2}\right)^{1 / 2}$ is not homogeneous.

We now present our main results.

Theorem 1. Let $\delta>0, L \in \mathbb{N}, L \geq[n / 2]+1, \beta>0$, and

$$
\mathcal{S}_{p}=\mathcal{S}_{p}(\beta)=\left(\frac{1}{p}-\frac{1}{2}\right) \max \{(\beta-2) n+2 \alpha n, 0\} .
$$

Assume that $\mu$ is a real-valued function of class $C^{N}(N \geq$ $L,[n / 2]+3)$ on $\mathbb{R}^{n} \backslash\{0\}$ which satisfies

$$
\begin{gathered}
\left|\partial^{\gamma} \mu(\xi)\right| \leq C_{\gamma}|\xi|^{\delta-|\gamma|}, \quad 0<|\xi| \leq 1, \quad|\gamma|=L, \\
\left|\partial^{\gamma} \mu(\xi)\right| \leq C_{\gamma}|\xi|^{\beta-|\gamma|}, \quad|\xi|>1,2 \leq|\gamma| \leq\left[\frac{n}{2}\right]+3 .
\end{gathered}
$$

Suppose also that $1 \leq p, q \leq \infty, s_{i} \in \mathbb{R}, \alpha \in[0,1]$, for $i=1,2$, and satisfies $s_{1}-s_{2} \geq\left|\mathcal{S}_{p}\right|$. Then one has

$$
\left\|e^{i \mu(D)} f\right\|_{M_{p, q}^{s_{2}, \alpha}} \leq C\|f\|_{M_{p, q}^{s_{1}, \alpha}}
$$

where the constant $C$ is independent of $f$.

Corollary 2. Let $\delta>0, L \in \mathbb{N}, L \geq[n / 2]+1, \theta>0, \lambda>0$, and

$$
\mathcal{S}_{p}(\beta)=\left(\frac{1}{p}-\frac{1}{2}\right) \max \{(\beta-2) n+2 \alpha n, 0\} .
$$

Assume that $\phi$ is a real-valued $C^{N}(\mathbb{R} \backslash\{0\})(N \geq L,[n / 2]+3)$ function satisfying

$$
\begin{gathered}
\left|\partial^{k} \phi(r)\right| \leq C_{k}|r|^{\delta-k}, \quad 0<|r| \leq 1, \quad k=L, \\
\left|\partial^{k} \phi(r)\right| \leq C_{k}|r|^{\theta-|k|}, \quad|r|>1, \quad 1 \leq|k| \leq\left[\frac{n}{2}\right]+3 .
\end{gathered}
$$

Let $h: \mathbb{R}^{n} \backslash\{0\} \rightarrow \mathbb{R} \backslash\{0\}$ be a smooth positive homogeneous function with degree $\lambda$. Suppose $1 \leq p, q \leq \infty, s_{i} \in \mathbb{R}, \alpha \in$ $[0,1]$ for $i=1,2$, and satisfies $s_{1}-s_{2} \geq\left|\mathcal{S}_{p}(\lambda \theta)\right|$. Then one has

$$
\left\|e^{i \phi(h(D))} f\right\|_{M_{p, q}^{s_{2}, \alpha}} \leq C\|f\|_{M_{p, q}^{s_{1}, \alpha}}
$$

where the constant $C$ is independent of $f$.

Theorem 3. Let $\delta>0, L \in \mathbb{N}, L \geq[n / 2]+1, \beta>0, \beta \neq 1$, $\lambda>0$, and

$$
\mathcal{S}_{p}=\mathcal{S}_{p}(\beta)=\left(\frac{1}{p}-\frac{1}{2}\right) \max \{(\beta-2) n+2 \alpha n, 0\} .
$$

Assume that $\phi$ is a real-valued $C^{N}\left(\mathbb{R}^{+}\right)(N \geq L,[n / 2]+3)$ function which satisfies

$$
\begin{gathered}
\left|\partial^{k} \phi(r)\right| \leq C_{k}|r|^{\delta-k}, \quad 0<|r| \leq 1, k=L, \\
\left|\partial^{k} \phi(r)\right| \leq C_{k}|r|^{\beta-|k|}, \quad|r|>1, \quad 1 \leq|k| \leq\left[\frac{n}{2}\right]+3, \\
\left|\partial^{k} \phi(r)\right| \sim|r|^{\beta-k}, \quad|r|>1, k=1,2 .
\end{gathered}
$$

Let $1 \leq p_{i}, q_{i} \leq \infty, s_{i} \in \mathbb{R}, \alpha \in[0,1]$ for $i=1,2$. Then

$$
\left\|e^{i \phi(|D|)} f\right\|_{M_{p_{2}, q_{2}}^{s_{2}, \alpha}} \leqslant\|f\|_{M_{p_{1}, q_{1}}^{s_{1}, \alpha}}
$$

holds for all $f$ if and only if

$$
\begin{gathered}
\frac{1}{p_{2}} \leq \frac{1}{p_{1}} \\
s_{2}-\frac{n \alpha}{p_{2}}+\max \left\{\delta_{p_{2}}, \mathcal{\delta}_{p_{1}^{\prime}}\right\}=s_{1}-\frac{n \alpha}{p_{1}}, \\
\frac{1}{q_{2}} \leq \frac{1}{q_{1}}
\end{gathered}
$$


or

$$
\begin{gathered}
\frac{1}{p_{2}} \leq \frac{1}{p_{1}}, \\
s_{2}-\frac{n \alpha}{p_{2}}+\max \left\{\delta_{p_{2}}, \mathcal{\delta}_{p_{1}^{\prime}}\right\}<s_{1}-\frac{n \alpha}{p_{1}}, \\
s_{2}-\frac{n \alpha}{p_{2}}+\max \left\{\delta_{p_{2}}, \mathcal{S}_{p_{1}^{\prime}}\right\}+\frac{n(1-\alpha)}{q_{2}} \\
<s_{1}-\frac{n \alpha}{p_{1}}+\frac{n(1-\alpha)}{q_{1}} .
\end{gathered}
$$

We list two examples to illustrate the assumptions in our theorems. First, the function $\mu(\xi)=\left(1+\sum_{j=1}^{n} \lambda_{j}|\xi|_{j}^{\lambda}\right)^{\theta}\left(\lambda_{j} \geq\right.$ $0, \lambda>0, \theta>0$ ) satisfies the assumptions in Theorem 1 and Corollary 2 for $\beta=\lambda \theta>0$, while $\mu(\xi)$ is not radial and not homogeneous. Another function is $\mu(\xi)=\mu(|\xi|)$ with $\mu(r)=\left(1+r^{\lambda}\right)^{\theta}(\lambda, \theta>0)$. This function satisfies the assumptions in Theorem 3 for $\beta=\lambda \theta, \lambda \theta \neq 1$. One may also observe that if $\beta=1$, there exists no $C^{2}\left(\mathbb{R}^{+}\right)$function $\phi(r)$, which satisfies the size condition (16). If the reader checks the main theorems in $[5,6]$, it is not difficult to see that our theorems are a substantial improvement and extension of the known results, even in the case $\alpha=0$.

The paper is organized as follows. In Section 2, we recall some definitions and basic properties. In Section 3, we obtain an improvement of results in $[5,6]$ by studying more general Fourier multipliers $e^{i \mu(D)}$, in which we do not need to assume lower order derivatives of $\mu(\xi)$ near 0 . This new results will be used to achieve a more general result for the boundedness of $e^{i \mu(D)}$ on spaces $M_{p, q}^{s, \alpha}$. In Section 4 , by assuming radial condition on $\mu(\xi)$, we deduce a dual estimate of $e^{i \phi(|D|)}$, and then we use the method in [14] to give a sharp result for the boundedness of $e^{i \phi(|D|)}$ between $M_{p_{1}, q_{1}}^{s_{1}, \alpha}$ and $M_{p_{2}, q_{2}}^{s_{2}, \alpha}$.

\section{Preliminaries}

We start this section by recalling some notations. Let $C$ be a positive constant that may depend on the indices $n, p_{i}, q_{i}, s_{i}, \alpha, \beta$. The notation $X \leqslant Y$ denotes the statement that $X \leq C Y$, the notation $X \sim Y$ means the statement $X \lesssim Y \lesssim X$, and the notation $X \simeq Y$ denotes the statement $X=C Y$. For a multi-index $k=\left(k_{1}, k_{2}, \ldots, k_{n}\right) \in \mathbb{Z}^{n}$, we denote $|k|_{\infty}:=\sup _{i=1,2, \ldots, n}\left|k_{i}\right|$ and $\langle k\rangle:=\left(1+|k|^{2}\right)^{1 / 2}$.

Let $\mathcal{S}:=\mathcal{S}\left(\mathbb{R}^{n}\right)$ be the Schwartz space and $\mathcal{S}^{\prime}:=\mathcal{S}^{\prime}\left(\mathbb{R}^{n}\right)$ the space of all tempered distributions. We define the Fourier transform $\mathscr{F} f$ and the inverse Fourier $\mathscr{F}^{-1} f$ of $f \in \mathcal{S}\left(\mathbb{R}^{n}\right)$ by

$$
\begin{gathered}
\mathscr{F} f(\xi)=\widehat{f}(\xi)=\int_{\mathbb{R}^{n}} f(x) e^{-2 \pi i x \cdot \xi} d x, \\
\mathscr{F}^{-1} f(x)=\widehat{f}(-x)=\int_{\mathbb{R}^{n}} f(\xi) e^{2 \pi i x \cdot \xi} d \xi .
\end{gathered}
$$

To describe the function spaces discussed in this note, we first give the partition of unity on frequency space for $\alpha \in[0,1)$. We suppose $c>0$ and $C>0$ are two appropriate constants and choose a Schwartz function sequence $\left\{\eta_{k}^{\alpha}\right\}_{k \in \mathbb{Z}^{n}}$ satisfying

$$
\begin{aligned}
&\left|\eta_{k}^{\alpha}(\xi)\right| \geq 0, \quad \text { if }\left|\xi-k\langle k\rangle^{\alpha /(1-\alpha)}\right|<c\langle k\rangle^{\alpha /(1-\alpha)} \operatorname{supp} \eta_{k}^{\alpha} \subset\left\{\xi:\left|\xi-k\langle k\rangle^{\alpha /(1-\alpha)}\right|<C\langle k\rangle^{\alpha /(1-\alpha)}\right\} \\
& \sum_{k \in \mathbb{Z}^{n}} \eta_{k}^{\alpha}(\xi) \equiv 1, \quad \forall \xi \in \mathbb{R}^{n} ; \\
&\left|\partial^{\gamma} \eta_{k}^{\alpha}(\xi)\right| \leq C_{|\alpha|}\langle k\rangle^{-\alpha|\gamma| /(1-\alpha)} \\
& \forall \xi \in \mathbb{R}^{n}, \quad \gamma \in\left(\mathbb{Z}^{+} \cup\{0\}\right)^{n}
\end{aligned}
$$

Then $\left\{\eta_{k}^{\alpha}(\xi)\right\}_{k \in \mathbb{Z}^{n}}$ constitutes a smooth decomposition of unity. The frequency decomposition operators associated with above function sequence can be defined by

$$
\square_{k}^{\alpha}:=\mathscr{F}^{-1} \eta_{k}^{\alpha} \mathscr{F}
$$

for $k \in \mathbb{Z}^{n}$. Let $1 \leq p, q \leq \infty, s \in \mathbb{R}$, and $\alpha \in[0,1)$; the $\alpha$-modulation space associated with above decomposition is defined by

$$
\begin{aligned}
M_{p, q}^{s, \alpha}\left(\mathbb{R}^{n}\right)= & \left\{f \in \mathcal{S}^{\prime}\left(\mathbb{R}^{n}\right):\|f\|_{M_{p, q}^{s, \alpha}\left(\mathbb{R}^{n}\right)}\right. \\
& \left.=\left(\sum_{k \in \mathbb{Z}^{n}}\langle k\rangle^{s q /(1-\alpha)}\left\|\square_{k}^{\alpha} f\right\|_{p}^{q}\right)^{1 / q}<\infty\right\}
\end{aligned}
$$

with the usual modifications when $q=\infty$. For the sake of simplicity, in this note, we always denote $M_{p, q}^{s}=M_{p, q}^{s, 0}$ and $\eta_{k}(\xi)=\eta_{k}^{0}(\xi)$.

We introduce the dyadic decomposition of $\mathbb{R}^{n}$ in order to define the Besov space. Let $\varphi(\xi)$ be a smooth bump function supported in the ball $\{\xi:|\xi|<3 / 2\}$ and be identically equal to 1 on the ball $\{\xi:|\xi| \leq 4 / 3\}$. We denote

$$
\psi(\xi)=\varphi(\xi)-\varphi(2 \xi),
$$

and a function sequence

$$
\begin{aligned}
& \psi_{j}(\xi)=\psi\left(2^{-j} \xi\right), \quad j \in \mathbb{N} \\
& \psi_{0}(\xi)=1-\sum_{j \in \mathbb{N}} \psi_{j}(\xi)=\varphi(\xi) .
\end{aligned}
$$

For all integers $j \in \mathbb{N}$, we define the Littlewood-Paley operators

$$
\begin{aligned}
& \widehat{\Delta_{j} f}=\psi_{j}(\xi) \widehat{f}(\xi), \\
& \widehat{\Delta_{0} f}=\varphi(\xi) f(\xi) .
\end{aligned}
$$

Let $1 \leq p, q \leq \infty$, and $s \in \mathbb{R}$. For $f \in \mathcal{S}^{\prime}$ we set the the (inhomogeneous) Besov space space norm by

$$
\|f\|_{B_{p, q}^{s}}=\left(\sum_{j=0}^{\infty} 2^{j s q}\left\|\Delta_{j} f\right\|_{L^{p}}^{q}\right)^{1 / q} .
$$


The (inhomogeneous) Besov space is the space of all tempered distributions $f$ for which the quantity $\|f\|_{B_{p, q}^{s}}$ is finite. We recall the following embedding results.

Lemma 4 (embedding $[12,13]$ ). Let $1 \leq p_{i}, q_{i} \leq \infty, s_{i} \in \mathbb{R}$, for $i=1,2, \alpha \in[0,1]$. If

$$
\begin{aligned}
\frac{1}{p_{2}} & \leq \frac{1}{p_{1}}, \\
s_{2}-\frac{n \alpha}{p_{2}} & =s_{1}-\frac{n \alpha}{p_{1}}, \\
\frac{1}{q_{2}} & \leq \frac{1}{q_{1}}
\end{aligned}
$$

or

$$
\begin{gathered}
\frac{1}{p_{2}} \leq \frac{1}{p_{1}}, \\
s_{2}-\frac{n \alpha}{p_{2}}<s_{1}-\frac{n \alpha}{p_{1}}, \\
s_{2}-\frac{n \alpha}{p_{2}}+\frac{n(1-\alpha)}{q_{2}} \\
<s_{1}-\frac{n \alpha}{p_{1}}+\frac{n(1-\alpha)}{q_{1}},
\end{gathered}
$$

one has

$$
M_{p_{1}, q_{1}}^{s_{1}, \alpha} \subseteq M_{p_{2}, q_{2}}^{s_{2}, \alpha}
$$

We also recall some results obtained in $[6,14]$, respectively.

Lemma 5 (see Lemma 3.2 in [6]). Let $\epsilon>0$ and let $\mu$ be $a$ $C^{[n / 2]+1}$ function on $\mathbb{R}^{n} \backslash\{0\}$ satisfying

$$
\left|\partial^{\gamma} \mu(\xi)\right| \leq C_{\gamma}|\xi|^{\epsilon-\gamma}
$$

for $|\gamma| \leq[n / 2]+1$. Then $\mathscr{F}^{-1}\left[\eta e^{i \mu(\xi)}\right] \in L^{1}\left(\mathbb{R}^{n}\right)$ for each $\eta \in$ $C_{c}^{\infty}\left(\mathbb{R}^{n}\right)$.

Lemma 6 (see Theorem 1.1 in [14]). Let $\beta>0, \epsilon>0$, and

$$
\delta_{p}=\delta_{p}(\beta)=\left(\frac{1}{p}-\frac{1}{2}\right) \max \{(\beta-2) n+2 \alpha n, 0\} .
$$

Assume that $\mu$ is a real-valued function of class $C^{[n / 2]+3}$ on $\mathbb{R}^{n} \backslash$ $\{0\}$ which satisfies

$$
\begin{aligned}
& \left|\partial^{\gamma} \mu(\xi)\right| \leq C_{\gamma}|\xi|^{\epsilon-|\gamma|}, \quad 0<|\xi| \leq 1,|\gamma| \leq\left[\frac{n}{2}\right]+1, \\
& \left|\partial^{\gamma} \mu(\xi)\right| \leq C_{\gamma}|\xi|^{\beta-|\gamma|}, \quad|\xi|>1,2 \leq|\gamma| \leq\left[\frac{n}{2}\right]+3 .
\end{aligned}
$$

Suppose also that $1 \leq p, q \leq \infty, s_{i} \in \mathbb{R}, \alpha \in[0,1]$, for $i=1,2$, and they satisfy $s_{1}-s_{2} \geq\left|\mathcal{S}_{p}\right|$. Then we have

$$
\left\|e^{i \mu(D)} f\right\|_{M_{p, q}^{s_{2}, \alpha}} \leq C\|f\|_{M_{p, q}^{s_{1}, \alpha}}
$$

where the constant $C$ is independent of $f$.

\section{Sufficient Condition of the Boundedness of $e^{i \mu(D)}$}

The goal of this section is to prove Theorem 1 and Corollary 2. We will start with the following derivative lemma for showing that the lower order derivative near 0 does not interrupt the boundedness of $e^{i \mu(D)}$ on $\alpha$-modulation spaces.

Lemma 7 (derivative lemma). Let $L \in \mathbb{Z}, L \geq 0, \delta>0$. Suppose that $m \in C^{L}\left(\mathbb{R}^{n} \backslash\{0\}\right)$ satisfying

$$
\left|\partial^{\gamma} m(\xi)\right| \leq C_{\gamma}|\xi|^{\delta-|\gamma|}, \quad 0<|\xi| \leq 1, \quad|\gamma|=L .
$$

Then the limit

$$
a=\lim _{\xi \rightarrow 0} m(\xi)=m(0)
$$

exists, and for any $\epsilon \in(0,1) \cap(0, \delta]$, we have

$$
\left|\partial^{\gamma}(m-a)(\xi)\right| \leq C_{\gamma}|\xi|^{\epsilon-|\gamma|}, \quad 0<|\xi| \leq 1,|\gamma| \leq L .
$$

Proof. We will state the proof for the cases $L=0,1,2$; the other cases can be deduced by a similar argument and an easy induction.

For $L=0$, one can observe directly that $a=0=$ $\lim _{\xi \rightarrow 0} m(\xi)$.

For $L=1$, fix $\epsilon \in(0,1) \cap(0, \delta)$. For any $\xi_{1}, \xi_{2} \in B(0,1)$, $\left|\xi_{1}\right| \leq\left|\xi_{2}\right|$, we can find a simple piecewise smooth curve

$$
\Gamma:[0, l] \longrightarrow \mathbb{R}^{n},
$$

which is jointed by two curves $\Gamma_{1}$ and $\Gamma_{2}$ with length $l=|\Gamma|$, where $\Gamma_{1}$ is the straight line connecting points $\xi_{1}$ and $\left(\left|\xi_{2}\right| /\right.$ $\left.\left|\xi_{1}\right|\right) \xi_{1}$ and $\Gamma_{2}$ is shortest curve on the great circle connecting points $\left(\left|\xi_{2}\right| /\left|\xi_{1}\right|\right) \cdot \xi_{1}$ and $\xi_{2}$, such that

$$
\begin{gathered}
\Gamma(0)=\xi_{1}, \quad \Gamma\left(\left|\xi_{2}\right|-\left|\xi_{1}\right|\right)=\frac{\left|\xi_{2}\right|}{\left|\xi_{1}\right|} \xi_{1}, \quad \Gamma(l)=\xi_{2}, \\
\left|\Gamma^{\prime}(t)\right|=1
\end{gathered}
$$

for all $t \in[0, l]$. We have

$$
\begin{aligned}
\mid m & \left(\xi_{2}\right)-m\left(\xi_{1}\right) \mid \\
= & |m(\Gamma(l))-m(\Gamma(0))| \\
\leq & \left|m(\Gamma(l))-m\left(\Gamma\left(\left|\xi_{2}\right|-\left|\xi_{1}\right|\right)\right)\right| \\
& \quad+\left|m\left(\Gamma\left(\left|\xi_{2}\right|-\left|\xi_{1}\right|\right)\right)-m(\Gamma(0))\right|, \\
\left|m\left(\Gamma\left(\left|\xi_{2}\right|-\left|\xi_{1}\right|\right)\right)-m(\Gamma(0))\right| & \left|\int_{0}^{\left|\xi_{2}\right|-\left|\xi_{1}\right|}(\nabla m)(\Gamma(t)) \cdot \Gamma^{\prime}(t) d t\right| \\
= & \int_{0}^{\left|\xi_{2}\right|-\left|\xi_{1}\right|}|(\nabla m)(\Gamma(t))| d t \\
\lesssim & \int_{0}^{\left|\xi_{2}\right|-\left|\xi_{1}\right|}\left(\left|\xi_{1}\right|+t\right)^{\epsilon-1} d t \\
= & \frac{\left|\xi_{2}\right|^{\epsilon}-\left|\xi_{1}\right|^{\epsilon}}{\epsilon},
\end{aligned}
$$




$$
\begin{aligned}
\mid m & (\Gamma(l))-m\left(\Gamma\left(\left|\xi_{2}\right|-\left|\xi_{1}\right|\right)\right) \mid \\
& =\left|\int_{\left|\xi_{2}\right|-\left|\xi_{1}\right|}^{l}(\nabla m)(\Gamma(t)) \cdot \Gamma^{\prime}(t) d t\right| \\
& \lesssim \int_{\left|\xi_{2}\right|-\left|\xi_{1}\right|}^{l}|(\nabla m)(\Gamma(t))| d t \\
& \leqslant \int_{\left|\xi_{2}\right|-\left|\xi_{1}\right|}^{l}\left|\xi_{2}\right|^{\epsilon-1} d t \lesssim\left|\xi_{2}\right|^{\epsilon} .
\end{aligned}
$$

Hence,

$$
\left|m\left(\xi_{2}\right)-m\left(\xi_{1}\right)\right| \lesssim \frac{\left|\xi_{2}\right|^{\epsilon}-\left|\xi_{1}\right|^{\epsilon}}{\epsilon}+\left|\xi_{2}\right|^{\epsilon} \longrightarrow 0
$$

as $\xi_{1}, \xi_{2} \rightarrow 0$. So the limit

$$
a=\lim _{\xi \rightarrow 0} m(\xi)=m(0)
$$

exists and

$$
|m(\xi)-a|=\lim _{\eta \rightarrow 0}|m(\xi)-m(\eta)| \leq \frac{|\xi|^{\epsilon}}{\epsilon}+|\xi|^{\epsilon} \lesssim|\xi|^{\epsilon} .
$$

For $L=2$, fix $\epsilon \in(0,1) \cap(0, \delta)$. For any $\xi \in B(0,1)$, let $\Gamma$ be the straight line connecting $\xi$ and $\xi /|\xi|$, such that

$$
\begin{gathered}
\Gamma(0)=\xi, \quad \Gamma(1-|\xi|)=\frac{\xi}{|\xi|}, \\
\left|\Gamma^{\prime}(t)\right|=1 .
\end{gathered}
$$

For any $j=1,2, \ldots, n$, we have

$$
\begin{aligned}
& \left|\partial_{j} m(\xi)-\partial_{j} m\left(\frac{\xi}{|\xi|}\right)\right| \\
& \quad=\left|\left(\partial_{j} m\right)(\Gamma(1-|\xi|))-\left(\partial_{j} m\right)(\Gamma(0))\right| \\
& \quad=\left|\int_{0}^{1-|\xi|}\left(\nabla \partial_{j} m\right)(\Gamma(t)) \cdot \Gamma^{\prime}(t) d t\right| \\
& \quad \lesssim \int_{0}^{1-|\xi|}\left|\left(\nabla \partial_{j} m\right)(\Gamma(t))\right| d t \\
& \quad \leq \int_{0}^{1-|\xi|}(|\xi|+t)^{\epsilon-2} d t \\
& \quad=\frac{1-|\xi|^{\epsilon-1}}{\epsilon-1} .
\end{aligned}
$$

It follows that

$$
\begin{aligned}
\left|\partial_{j} m(\xi)\right| & \leq\left|\partial_{j} m(\xi)-\partial_{j} m\left(\frac{\xi}{|\xi|}\right)\right|+\left|\partial_{j} m\left(\frac{\xi}{|\xi|}\right)\right| \\
& \leq \frac{1-|\xi|^{\epsilon-1}}{\epsilon-1}+1 \lesssim|\xi|^{\epsilon-1} .
\end{aligned}
$$

Now we finish the proof by repeating the $L=1$ case.
We are in a position to give the proof of Theorem 1.

Proof of Theorem 1. In virtue of the above lemma, since

$$
\left|\partial^{\gamma} \mu(\xi)\right| \leq C_{\gamma}|\xi|^{\delta-|\gamma|}, \quad 0<|\xi| \leq 1,|\gamma|=L,
$$

and $L \geq[n / 2]+1$, for fixed $\epsilon \in(0,1) \cap(0, \delta]$, we have

$$
\left|\partial^{\gamma}(\mu-\mu(0))(\xi)\right| \leq C_{\gamma}|\xi|^{\epsilon-|\gamma|}, \quad 0<|\xi| \leq 1
$$

for any $|\gamma| \leq[n / 2]+1$.

Using Lemma 5, we know that

$$
\mathscr{F}^{-1}\left[\eta e^{i \mu(\xi)}\right]=\mathscr{F}^{-1}\left[\eta e^{i(\mu-\mu(0))(\xi)}\right] \in L^{1} .
$$

Finally, we use Lemma 6 to complete the proof.

Proof of Corollary 2. By the assumptions of Corollary 2, we can use Lemma 7 to deduce that

$$
\left|\partial^{k}(\phi-\phi(0))(r)\right| \leq C_{k}|r|^{\epsilon-k}, \quad 0<|r| \leq 1
$$

for fixed $\epsilon \in(0,1) \cap(0, \delta]$ and any $k \leq[n / 2]+1$. The assumption of $h(\xi)$ implies that

$$
|h(\xi)| \sim|\xi|^{\lambda} .
$$

It follows then

$$
\left|\partial^{\gamma}(\phi \circ h)(\xi)\right| \lesssim|\xi|^{\lambda \epsilon-|\gamma|}, \quad 0<|\xi| \leq 1,
$$

for any $|\gamma|=[n / 2]+1$.

On the other hand, one can deduce that

$$
\left|\partial^{\gamma}(\phi \circ h)(\xi)\right| \leq|\xi|^{\lambda \theta-|\gamma|}, \quad|\xi| \geq 1,
$$

for $2 \leq|\gamma| \leq[n / 2]+3$.

Finally, the conclusion is deduced by Theorem 1 .

\section{Sharpness of the Conditions for the Boundenness of $e^{i \phi(|\xi|)}$}

In this section, we give the proof of Theorem 3 . The key point is that we can obtain a dual estimate for $e^{i \phi(|\xi|)}$ under some size condition on $\phi$. By combining the dual estimate with Theorem 1, we get the simultaneous asymptotic estimates of $\|f\|_{M_{p, q}^{s, \alpha}}$ and $\left\|e^{i \mu(D)}\right\|_{M_{p, q}^{s, \alpha}}$. Then the proof can be finished by the method in [14]. We first start with the dual estimate on Besov spaces.

Lemma 8 (dual estimate for $B_{p, q}^{s}$ ). Suppose $\beta>0, \beta \neq 1$. Assume that $\phi$ is a real-valued $C^{[n / 2]+3}$ function which satisfies the assumptions of Theorem 3 . Then one has

$$
\left\|\mathscr{F}^{-1}\left[\psi_{j}(\xi) e^{i \phi(\xi))}\right]\right\|_{L^{\infty}} \leq C 2^{j(\beta-2)(-n / 2)}
$$

for all $j \in \mathbb{N}$. 
Proof. Using the change of variables, we have

$$
\left\|\mathscr{F}^{-1}\left[\psi_{j}(\xi) e^{i \phi(|\xi|)}\right]\right\|_{L^{\infty}}=2^{j n}\left\|\mathscr{F}^{-1}\left[\psi(\xi) e^{i \phi\left(2^{j}|\xi|\right)}\right]\right\|_{L^{\infty}} .
$$

Use the polar coordinates,

$$
\begin{aligned}
\mathscr{F}^{-1} & {\left[\psi(\xi) e^{i \phi\left(2^{j}|\xi|\right)}\right](x) } \\
& =\int_{\mathbb{R}^{n}} \psi(\xi) e^{i \phi\left(2^{j}|\xi|\right)} e^{2 \pi i x \cdot \xi} d \xi \\
& =\int_{0}^{\infty} \psi(r) e^{i \phi\left(2^{j} r\right)} r^{n-1} \int_{S^{n-1}} e^{2 \pi i r x \cdot \xi^{\prime}} d \sigma\left(\xi^{\prime}\right) d r \\
& =\int_{0}^{\infty} \psi(r) e^{i \phi\left(2^{j} r\right)} r^{n-1} \widehat{d \sigma}(-r x) d r .
\end{aligned}
$$

Recall that the Fourier transform of the area measure satisfies

$$
\begin{aligned}
\widehat{d \sigma}(-r x)= & C \frac{e^{2 \pi i r|x|}}{|r x|^{(n-1) / 2}} \\
& +C \frac{e^{-2 \pi i r|x|}}{|r x|^{(n-1) / 2}}+O\left(|r x|^{-n / 2}\right) .
\end{aligned}
$$

The support of $\psi$ yields $1 /|r x|^{(n-1) / 2} \lesssim 1 /|x|^{(n-1) / 2}$ and $O\left(|r x|^{-n / 2}\right) \lesssim|x|^{-n / 2}$.

For the case $|x| \gtrsim 2^{j \beta}$, we only need to show that

$$
\left|\int_{0}^{\infty} \psi(r) e^{i\left[\phi\left(2^{j} r\right) \pm 2 \pi i r|x|\right]} r^{n-1} d r\right| \lesssim 2^{-j \beta(n / 2)},
$$

which is a direct conclusion by the fact that

$$
\frac{d^{2}\left(\phi\left(2^{j} r\right) \pm 2 \pi i r|x|\right)}{d r^{2}} \gtrsim 2^{j \beta}
$$

and the Van der Corput lemma.

For the case that $|x| \lesssim 2^{j \beta}$, we define

$$
L:=\frac{\sum_{j=1}^{n} \xi_{j} \partial_{j}}{i\left[2^{j}|\xi| \phi^{\prime}\left(2^{j}|\xi|\right)+\sum_{j=1}^{n} 2 \pi x_{j} \xi_{j}\right]}
$$

and notice that

$$
L\left(e^{i\left[\phi\left(2^{j} r\right) \pm 2 \pi i r|x|\right]}\right)=e^{i\left[\phi\left(2^{j} r\right) \pm 2 \pi i r|x|\right]} .
$$

Then the inequality

$$
\left|\int_{\mathbb{R}^{n}} \psi(\xi) e^{i \phi\left(2^{j}|\xi|\right)} e^{2 \pi i x \cdot \xi} d \xi\right| \lesssim\left(2^{j \beta}\right)^{-n / 2}
$$

follows by an integration by parts.

Hence, for all $j \in \mathbb{N}$,

$$
\left\|F^{-1}\left[\psi_{j}(\xi) e^{i \phi(|\xi|)}\right]\right\|_{L^{\infty}} \lesssim 2^{j(B-2)(-n / 2)} .
$$

Lemma 9 (dual estimate for $M_{p, q}^{s, \alpha}, \alpha \in[0,1)$ ). Suppose $\beta>0$, $\beta \neq 1$. Assume that $\phi$ is a real-valued $C^{[n / 2]+3}$ function satisfying the assumptions of Theorem 3. Then there exists a sufficiently large constant $R$ such that

$$
\left\|\mathscr{F}^{-1}\left[\eta_{k}^{\alpha}(\xi) e^{i \phi(|\xi|)}\right]\right\|_{L^{\infty}} \leq C\langle k\rangle^{((\beta-2) /(1-\alpha))(-n / 2)},
$$

for all $k \in \mathbb{Z}^{n}$ with $\langle k\rangle^{\alpha /(1-\alpha)} k \in \mathbb{R}^{n} \backslash B(0, R)$, where the constant $C$ is independent of $k$.

Proof. For sufficiently large $k$, we use estimate on some $\Delta_{j}$ to estimate $\square_{k}^{\alpha}$. Choose a $j$ satisfying $\langle k\rangle^{1 /(1-\alpha)} \sim 2^{j}$. An easy computation shows that

$$
\begin{aligned}
& \left\|\mathscr{F}^{-1}\left[\eta_{k}^{\alpha}(\xi) e^{i \phi(|\xi|)}\right]\right\|_{L^{\infty}} \\
& \quad \lesssim\left\|\mathscr{F}^{-1}\left[\psi_{j}(\xi) \eta_{k}^{\alpha}(\xi) e^{i \phi(|\xi|)}\right]\right\|_{L^{\infty}} \\
& \quad \lesssim\left\|\mathscr{F}^{-1}\left[\psi_{j}(\xi) e^{i \phi(|\xi|)}\right]\right\|_{L^{\infty}} \\
& \quad \lesssim 2^{j(\beta-2)(-n / 2)} \sim\langle k\rangle^{((\beta-2) /(1-\alpha))(-n / 2)} .
\end{aligned}
$$

Now, we give the asymptotic estimates of $\left\|e^{i \phi(|D|)} f\right\|_{M_{p, q}^{s, \alpha}}$ and $\|f\|_{M_{p, q}^{s, \alpha}}$. These results can be verified by the same methods in [14].

Lemma 10 (asymptotic estimates of $\left\|e^{i \phi(|D|)} f\right\|_{M_{p, q}^{s, \alpha}}(\alpha \in[0$, $1))$ ). Suppose $\beta>0, \beta \neq 1$. Assume that $\phi$ is a real-valued function which satisfies the assumptions of Theorem 3. we can find function sequences $\left\{f_{\lambda}\right\},\left\{h_{k}\right\}$, and $\left\{g_{N}\right\}$ such that

$$
\begin{gathered}
\left\|e^{i \phi(|D|)} f_{\lambda}\right\|_{M_{p, q}^{s, \alpha}} \sim \lambda^{n(1-(1 / p))} \quad \text { as } \lambda \longrightarrow 0, \\
\left\|e^{i \phi(|D|)} h_{k}\right\|_{M_{p, q}^{s, \alpha}} \sim\langle k\rangle^{\left(s+\mathcal{S}_{p}+\alpha n(1-1 / p)\right) /(1-\alpha)} \quad \text { as }|k| \longrightarrow \infty \\
\left\|e^{i \phi(|D|)} g_{N}\right\|_{M_{p, q}^{s, \alpha}} \sim N^{\left(\left(s+\mathcal{S}_{p}+\alpha n(1-1 / p)\right) /(1-\alpha)\right)+(n / q)} \\
\text { as } N \longrightarrow \infty, \quad s-\frac{n \alpha}{p}+\mathcal{S}_{p}+\frac{n(1-\alpha)}{q}>-n \alpha, \\
\left\|e^{i \phi(|D|)} g_{N}\right\|_{M_{p, q}^{s, \alpha}} \sim(\ln N)^{1 / q} \quad \text { as } N \longrightarrow \infty \\
s-\frac{n \alpha}{p}+\mathcal{S}_{p}+\frac{n(1-\alpha)}{q}=-n \alpha .
\end{gathered}
$$

Moreover, we can easily verify that

$$
\begin{gathered}
\left\|f_{\lambda}\right\|_{M_{p, q}^{s, \alpha}} \sim \lambda^{n(1-(1 / p))} \quad \text { as } \lambda \longrightarrow 0, \\
\left\|h_{k}\right\|_{M_{p, q}^{s, \alpha}} \sim\langle k\rangle^{(s+\alpha n(1-1 / p)) /(1-\alpha)} \quad \text { as }|k| \longrightarrow \infty, \\
\left\|g_{N}\right\|_{M_{p, q}^{s, \alpha}} \sim N^{((s+\alpha n(1-1 / p) /(1-\alpha))+(n / q)} \quad \text { as } N \longrightarrow \infty, \\
s-\frac{n \alpha}{p}+\frac{n(1-\alpha)}{q}>-n \alpha,
\end{gathered}
$$




$$
\begin{gathered}
\left\|g_{N}\right\|_{M_{p, q}^{s, \alpha}} \sim(\ln N)^{1 / q} \text { as } N \longrightarrow \infty \\
s-\frac{n \alpha}{p}+\frac{n(1-\alpha)}{q}=-n \alpha .
\end{gathered}
$$

Proof. In the case that $0<\beta \leq \Lambda_{\alpha}$ is trivial, we suppose that $\beta \geq \Lambda_{\alpha}$ in this proof. We only show the proof of (66).

Denote by $\widehat{h}(\xi)$ a $C_{c}^{\infty}\left(\mathbb{R}^{n}\right)$ function with sufficiently small support near zero. Let

$$
\widehat{f_{\lambda}}=\widehat{h}\left(\frac{\xi}{\lambda}\right), \quad \lambda \geq 1
$$

Using Theorem 1, we deduce that

$$
\left\|f_{\lambda}\right\|_{M_{p, q}^{s-\left|\delta_{p}\right|}, \alpha} \leqslant\left\|e^{i \phi(|D|)} f_{\lambda}\right\|_{M_{p, q}^{s, \alpha}} \lesssim\left\|f_{\lambda}\right\|_{M_{p, q}^{s+\left|\delta_{p}\right|}, \alpha} .
$$

Notice that

$$
\begin{aligned}
\left\|f_{\lambda}\right\|_{M_{p, q}^{s s \mid \delta p}, \alpha} & =\left\|f_{\lambda}\right\|_{M_{p, q}^{s+\left|\delta_{p}\right|, \alpha}}=\left\|\square_{0}^{\alpha} f_{\lambda}\right\|_{L^{p}} \\
& =\lambda^{n}\|h(\lambda x)\|_{L^{p}}=\lambda^{n(1-(1 / p))}\|h\|_{L^{p}} ;
\end{aligned}
$$

so we have

$$
\left\|e^{i \phi(|D|)} f_{\lambda}\right\|_{M_{p, q}^{s, \alpha}} \sim \lambda^{n(1-(1 / p))} \quad \text { as } \lambda \longrightarrow 0 .
$$

For the second equation, we denote

$$
\widehat{h}_{k}(\xi)=\widehat{h}\left(\frac{\xi-\langle k\rangle^{\alpha /(1-\alpha)} k}{\langle k\rangle^{\alpha /(1-\alpha)}}\right)
$$

and let $\widehat{f}_{k}(\xi)=\widehat{h}_{k}(\xi)$, where $k \in \mathbb{Z}^{n}$ with $\langle k\rangle^{\alpha /(1-\alpha)} k \in \Gamma \backslash$ $B(0, R)$. that

If $1 \leq p \leq 2$, we use Theorem 1 and Lemma 9 to deduce

$$
\left\|f_{k}\right\|_{M_{p^{\prime}, q}^{s+(1 / p-1 / 2)(\beta-2) n, \alpha}} \leqslant\left\|e^{i \phi(|D|)} f_{k}\right\|_{M_{p, q}^{s, \alpha}} \leqslant\left\|f_{k}\right\|_{M_{p, q}^{s+\delta_{p}, \alpha}} .
$$

If $2 \leq p \leq \infty$, we deduce that

$$
\left\|f_{k}\right\|_{M_{p, q}^{s+\delta_{p}, \alpha}} \lesssim\left\|e^{i \phi(|D|)} f_{k}\right\|_{M_{p, q}^{s, \alpha}} \lesssim\left\|f_{k}\right\|_{M_{p^{\prime}, q}^{s+(1 / p-1 / 2)(\beta-2) n, \alpha} .} .
$$

By a direct calculation, we obtain

$$
\begin{aligned}
\left\|f_{k}\right\|_{M_{p, q}^{s+\delta_{p}, \alpha}} & =\langle k\rangle^{\left(s+\mathcal{\delta}_{p}\right) /(1-\alpha)}\left\|h_{k}\right\|_{L^{p}} \\
& =\langle k\rangle^{\left(s+\mathcal{\delta}_{p}+\alpha n(1-1 / p)\right) /(1-\alpha)}\|h\|_{L^{p}}, \\
\left\|f_{k}\right\|_{M_{p^{\prime}, q}^{s+(1 / p-1 / 2)(\beta-2) n, \alpha}} & \\
= & \langle k\rangle^{(s+(1 / p-1 / 2)(\beta-2) n) /(1-\alpha)}\left\|h_{k}\right\|_{L^{p^{\prime}}} \\
& =\langle k\rangle^{\left(s+\mathcal{\delta}_{p}+\alpha n(1-1 / p)\right) /(1-\alpha)}\|h\|_{L^{p^{\prime}}} .
\end{aligned}
$$

Then the asymptotic formula follows.
For the third and fourth asymptotic formulas, let

$$
\widehat{f}_{N}(\xi)=\sum_{k \in A_{N}} \widehat{h}_{k}(\xi)
$$

where $A_{N}=\left\{k \in \mathbb{Z}^{n}:|k| \leq N,\langle k\rangle^{\alpha /(1-\alpha)} k \in \mathbb{R}^{n} \backslash B(0, R)\right\}$. By a direct calculation, we obtain

$$
\begin{aligned}
\left\|f_{N}\right\|_{M_{p, q}^{s+\delta_{p}, \alpha}} & \simeq\left(\sum_{k \in A_{N}}\langle k\rangle^{\left(\left(s+\delta_{p}+\alpha n(1-1 / p)\right) /(1-\alpha)\right) q}\right)^{1 / q} \\
& \simeq\left\|f_{N}\right\|_{\substack{p^{\prime}, q \\
s+(1 / p-1 / 2)(\beta-2) n, \alpha}}
\end{aligned}
$$

As above, Theorem 1 and Lemma 9 yield

$$
\left\|e^{i \phi(|D|)} f_{N}\right\|_{M_{p, q}^{s, \alpha}} \sim\left(\sum_{k \in A_{N}}\langle k\rangle^{\left(\left(s+\mathcal{S}_{p}+\alpha n(1-1 / p)\right) /(1-\alpha)\right) q}\right)^{1 / q} .
$$

Finally, the claim follows by

$$
\begin{gathered}
\left(\sum_{k \in A_{N}}\langle k\rangle^{\left(\left(s+\mathcal{S}_{p}+\alpha n(1-1 / p)\right) /(1-\alpha)\right) q}\right)^{1 / q} \\
\sim N^{\left(\left(s+\mathcal{S}_{p}+\alpha n(1-1 / p)\right) /(1-\alpha)\right)+(n / q)}
\end{gathered}
$$

for $s-(n \alpha / p)+\mathcal{S}_{p}+(n(1-\alpha) / q)>-n \alpha$, and

$$
\left(\sum_{k \in A_{N}}\langle k\rangle^{\left(\left(s+\delta_{p}+\alpha n(1-1 / p)\right) /(1-\alpha)\right) q}\right)^{1 / q} \sim(\ln N)^{1 / q}
$$

for $s-(n \alpha / p)+\mathcal{S}_{p}+(n(1-\alpha) / q)=-n \alpha$.

For the case $\alpha=1$, we have the following lemma; since its proof is similar to that of Lemma 10, we leave the detail to the reader.

Lemma 11 (asymptotic estimates of $\left\|e^{i \phi(|D|)} f\right\|_{B_{p, q}^{s}}$ ). Suppose $\beta>0, \beta \neq 1$. Assume that $\phi$ is a real-valued function which satisfies the assumptions of Theorem 3 . We can find function sequences $\left\{f_{\lambda}\right\},\left\{h_{k}\right\}$, and $\left\{g_{N}\right\}$ such that

$$
\begin{gathered}
\left\|e^{i \phi(|D|)} f_{\lambda}\right\|_{B_{p, q}^{s}} \sim \lambda^{n(1-(1 / p))} \quad \text { as } \lambda \longrightarrow 0, \\
\left\|e^{i \phi(|D|)} h_{j}\right\|_{B_{p, q}^{s}} \sim 2^{j\left[s+\mathcal{S}_{p}+n(1-1 / p)\right]} \quad \text { as }|j| \longrightarrow \infty, \\
\left\|e^{i \phi(|D|)} g_{N}\right\|_{B_{p, q}^{s}} \sim 2^{N\left[s+\mathcal{S}_{p}+n(1-1 / p)\right]} \quad \text { as } N \longrightarrow \infty, \\
s-\frac{n}{p}+\mathcal{S}_{p}>-n, \\
\left\|e^{i \phi(|D|)} g_{N}\right\|_{B_{p, q}^{s}} \sim N^{1 / q} \quad \text { as } N \longrightarrow \infty, \\
s-\frac{n}{p}+\mathcal{S}_{p}=-n .
\end{gathered}
$$


Moreover, we can easily verify that

$$
\begin{gathered}
\left\|f_{\lambda}\right\|_{B_{p, q}^{s}} \sim \lambda^{n(1-(1 / p))} \quad \text { as } \lambda \longrightarrow 0, \\
\left\|h_{j}\right\|_{B_{p, q}^{s}} \sim 2^{j[s+n(1-1 / p)]} \quad \text { as }|j| \longrightarrow \infty, \\
\left\|g_{N}\right\|_{B_{p, q}^{s}} \sim 2^{N[s+n(1-1 / p)]} \quad \text { as } N \longrightarrow \infty, \\
s-\frac{n}{p}>-n, \\
\left\|g_{N}\right\|_{B_{p, q}^{s}} \sim N^{1 / q} \quad \text { as } N \longrightarrow \infty, \\
s-\frac{n}{p}=-n .
\end{gathered}
$$

Now, we give the sketch of proof for Theorem 3.

Proof of Theorem 3. As in [14], if

$$
\left\|e^{i \phi(|D|)} f\right\|_{M_{p_{2}, q_{2}}^{s_{2}, \alpha}} \lesssim\|f\|_{M_{p_{1}, q_{1}}^{s_{1}, \alpha}}
$$

holds for all $f$, we can use Lemmas 10 and 11 to obtain (18) or (19).

On the other hand, if (18) or (19) holds, we can use the embedding lemma (Lemma 4), the dual estimates (Lemma 8 and Lemma 9), and Theorem 1 to obtain the boundedness of $e^{i \phi(|D|)}$ from $M_{p_{1}, q_{1}}^{s_{1}, \alpha_{1}}$ to $M_{p_{2}, q_{2}}^{s_{2}, \alpha_{2}}$.

\section{Conflict of Interests}

The authors declare that there is no conflict of interests regarding the publication of this paper.

\section{Acknowledgments}

The authors would like to thank the anonymous referee for helpful comments. This work is partially supported by the NSF of China (Grant no. 11271330).

\section{References}

[1] H. G. Feichtinger, "Modulation spaces on locally compact Abelian group," Tech. Rep., University of Vienna, 1983.

[2] P. Grobner, Banachraume Glatter Funktionen and Zerlegungsmethoden [Ph.D. thesis], University of Vienna, 1992.

[3] L. Li, "Some remarks on a multiplier theorem of Hormander," Mathematica Applicata, vol. 15, pp. 152-154, 2002.

[4] T. Mizuhara, "On Fourier multipliers of homogeneous Besov spaces," Mathematische Nachrichten, vol. 133, pp. 155-161, 1987.

[5] Á. Bényi, K. A. Gröchenig, and L. G. Rogers, "Unimodular Fourier multipliers for modulation spaces," Journal of Functional Analysis, vol. 246, no. 2, pp. 366-384, 2007.

[6] A. Miyachi, F. Nicola, S. Rivetti, and N. Tomita, "Estimates for unimodular Fourier multipliers on modulation spaces," Proceedings of the American Mathematical Society, vol. 137, no. 11, pp. 3869-3883, 2009.
[7] Á. Bényi, K. Gröchenig, C. Heil, and K. Okoudjou, "Modulation spaces and a class of bounded multilinear pseudodifferential operators," Journal of Operator Theory, vol. 54, no. 2, pp. 387399, 2005.

[8] J. Chen, D. Fan, and L. Sun, "Asymptotic estimates for unimodular Fourier multipliers on modulation spaces," Discrete and Continuous Dynamical Systems A, vol. 32, no. 2, pp. 467-485, 2012.

[9] Q. Deng, Y. Ding, and L. Sun, "Estimate for generalized unimodular multipliers on modulation spaces," Nonlinear Analysis: Theory, Methods and Applications, vol. 85, pp. 78-92, 2013.

[10] W. Guo and J. Chen, "Strichartz estimates on $\alpha$-modulation spaces," Electronic Journal of Differential Equations, no. 118, pp. $1-13,2013$.

[11] H. G. Feichtinger and G. Narimani, "Fourier multipliers of classical modulation spaces," Applied and Computational Harmonic Analysis. Time-Frequency and Time-Scale Analysis, Wavelets, Numerical Algorithms, and Applications, vol. 21, no. 3, pp. 349359, 2006.

[12] H. Triebel, Theory of Function Spaces, vol. 78 of Monographs in Mathematics, Birkhäuser, Boston, Mass, USA, 1983.

[13] J. S. Han and B. X. Wang, " $\alpha$-modulation spaces (I) scaling, embedding and algebraic properties," http://www.arxiv.org/ abs/1108.0460.

[14] G. P. Zhao, J. C. Chen, D. S. Fan, and C. Guo, "Unimodular Fourier multipliers and complex interpolation spaces on $\alpha$ modulation spaces," submitted. 


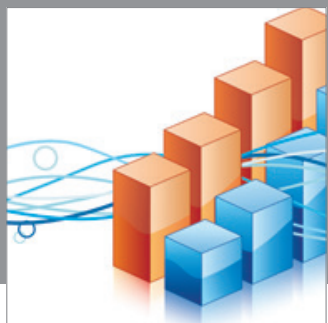

Advances in

Operations Research

mansans

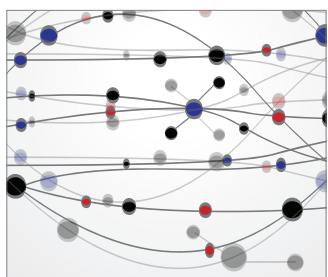

The Scientific World Journal
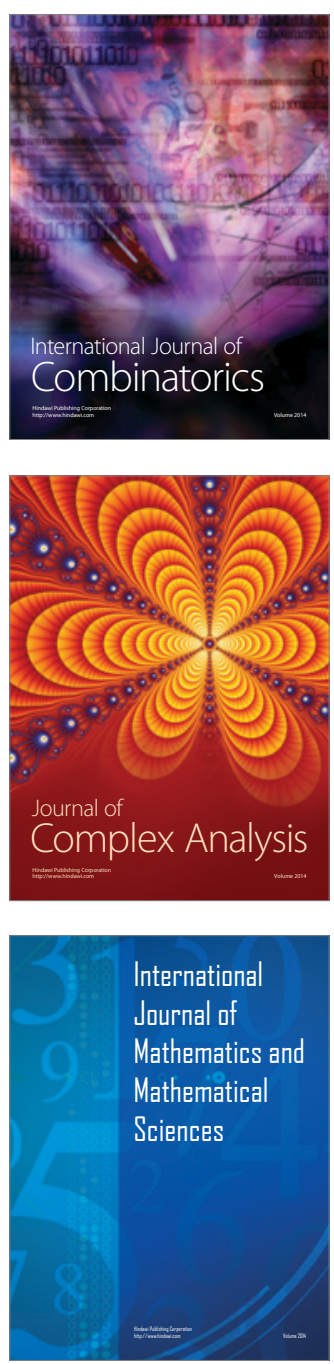
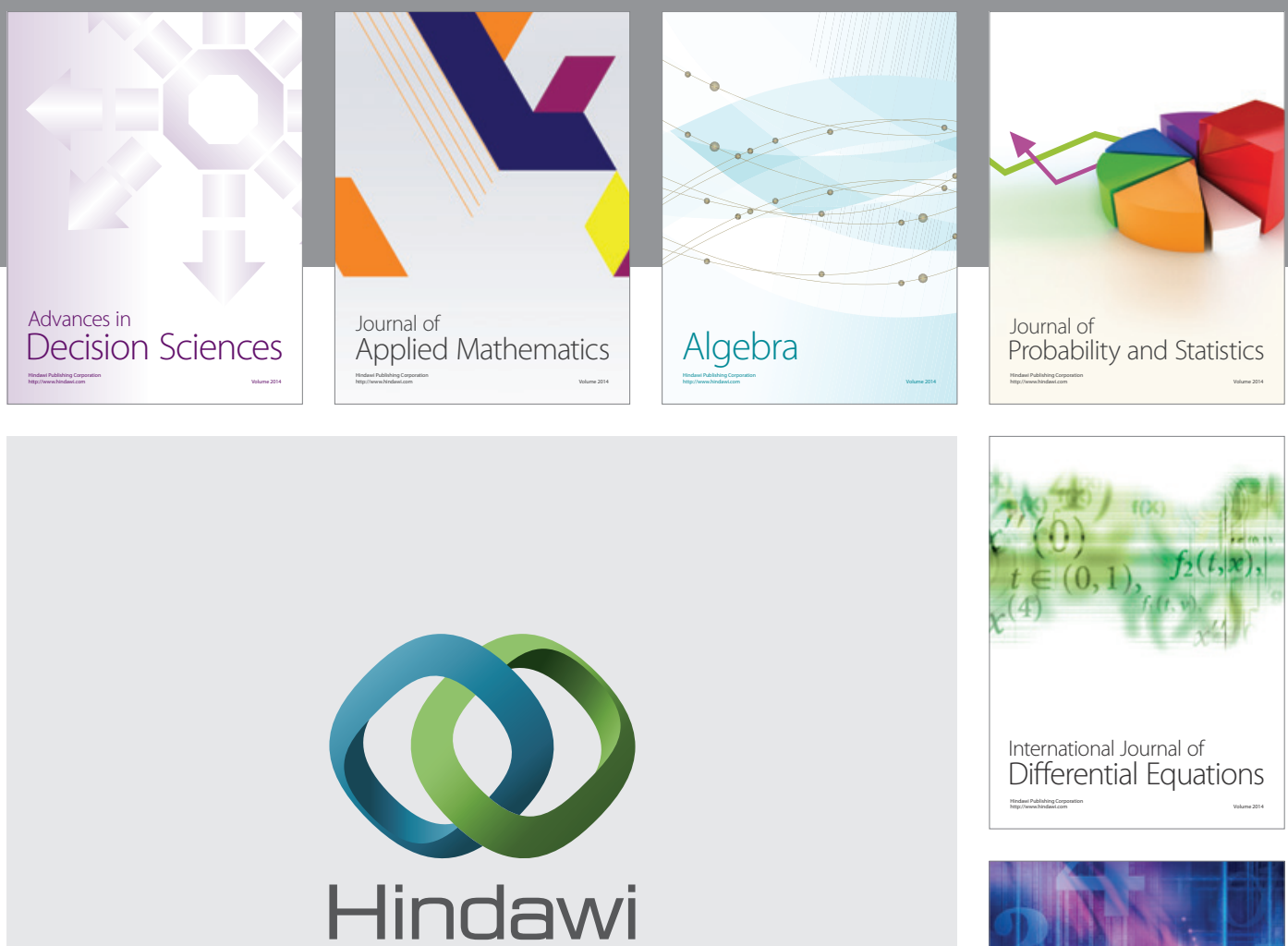

Submit your manuscripts at http://www.hindawi.com
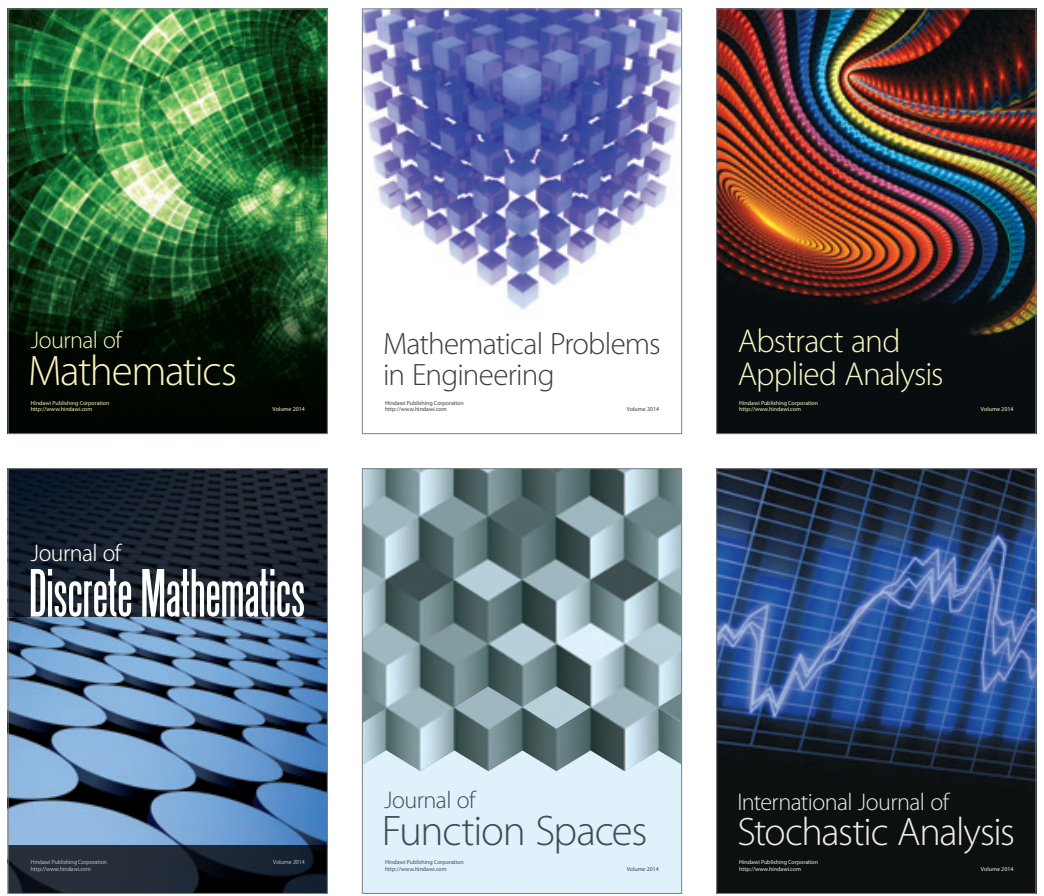

Journal of

Function Spaces

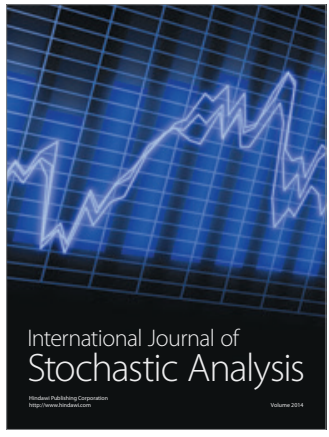

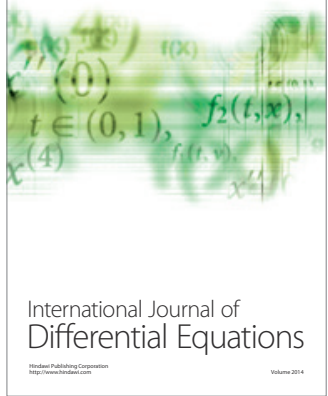
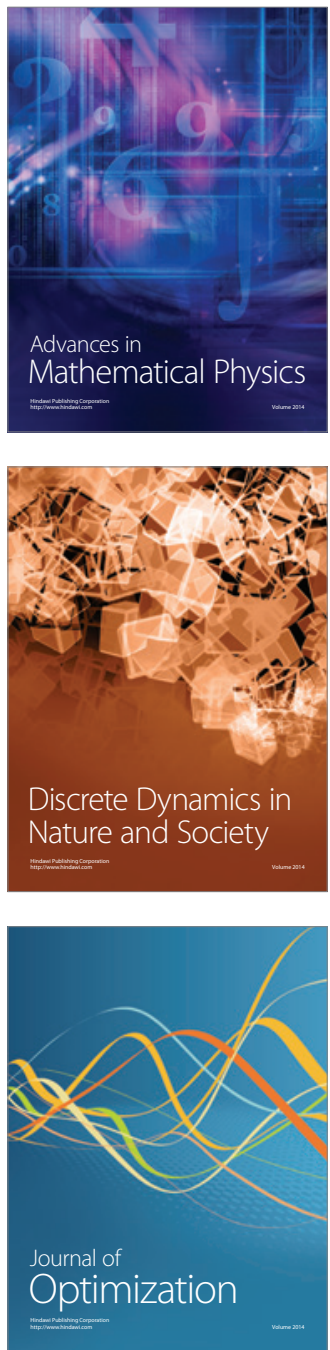